\title{
Wanted: a WhatsApp alternative for clinicians
}

In this article (BMJ 2018;360:k622, doi:10.1136/bmj.k622) we omitted an $\mathrm{n}$ from Helgi Johannsson's name. The implementation date of the General Data Protection Regulation was also incorrect. It comes into force in May 2018. 